\title{
Distribution of Somatostatin Immunoreactivity in the Human Dentate Gyrus
}

\author{
David G. Amaral,' Ricardo Insausti, ${ }^{1,2}$ and Michael J. Campbell ${ }^{3}$ \\ 1The Salk Institute for Biological Studies, San Diego, California 92138, 2Department of Anatomy, University of Navarra, \\ 31080 Pamplona, Spain, and ${ }^{3}$ Division of Preclinical Neuroscience and Endocrinology, Research Institute of Scripps Clinic, \\ La Jolla, California 92037
}

In previous immunohistochemical studies in the rat and monkey, a system of somatostatin-positive neurons and fibers was observed in the dentate gyrus of the hippocampal formation. In both species, somatostatin-immunoreactive cell bodies are located primarily in the deep or polymorphic layer of the dentate gyrus, and they give rise to a fiber system that terminates principally in the outer two-thirds of the molecular layer. In the present study, we employed the same antisera and staining procedures to determine whether the organization of the somatostatin system in the human dentate gyrus is similar to that seen in the rat and nonhuman primate. Sections of human postmortem brain material incubated with antisera directed against somatostatin $28_{1-12}$ (\$320) or somatostatin 28 (\$309) demonstrated a heterogeneous population of immunoreactive cells in the hilar region of the human dentate gyrus. Fiber staining was observed both in the hilar region and throughout the molecular layer, but the densest fiber and terminal plexus were observed in the outer two-thirds of the molecular layer. In addition, there were forms of somatostatin-immunoreactive profiles in the human sections that were not previously observed in the rat or monkey. Immunoreactive, grapelike clusters of apparently large, axonal varicosities were commonly observed, for example, as were dendritic profiles containing typical dendritic spines. In general, however, staining for somatostatin immunoreactivity in the human dentate gyrus presented a picture qualitatively similar to that observed in the rat and monkey.

Thus, immunohistochemical methods have allowed the analysis of a chemically defined neural system in the human brain that has been extensively studied in rat and monkey brains with both experimental and immunohistochemical methods. That the pattern of labeling in the human sections

Received Sept. 15, 1987; accepted Dec. 10, 1987.

This study would not have been possible without the enthusiastic support of several colleagues who have provided us with human postmortem brain material. We would like to express our appreciation to Drs. J. M. Martinez-Peñuela, T. Tuñon, and T. Urrutia of the Department of Pathology, Hospital of Navarra, Pamplona, Spain; Dr. Robert Nakamura and his staff at the Department of Pathology, Scripps Clinic and Research Foundation; and Dr. Joe Rogers of The Institute for Biogerontology Research, Sun City, Arizona. We would also like to thank Ms. Janet Weber and Mr. Casey Cox for their excellent technical assistance, and Mr. Kris Trulock for photographic processing. This work was supported by NIH Grants NS16980, MH4 1479, NIAAA Grants AA-07456 and AA-06420, the MacArthur Foundation, the Clayton Foundation for Research, California Division, and Grant FIS86/1129 from the Ministry of Health, Spain.

Correspondence should be addressed to Dr. David G. Amaral, The Salk Institute, P.O. Box 85800, San Diego, CA 92138.

Copyright (C) 1988 Society for Neuroscience $0270-6474 / 88 / 093306-11 \$ 02.00 / 0$ closely parallels that observed in the experimental animals provides support for the contention that immunohistochemical methods can reliably be employed to determine the normal neuroanatomical organization of the human brain. These methods may also be particularly applicable for the analysis of pathological brain conditions. In particular, alterations of the hippocampal somatostatin system have been associated with both Alzheimer's disease and temporal lobe epilepsy. It would be of interest, therefore, to apply immunohistochemical procedures to determine whether the anatomical organization of the human hippocampal somatostatin system is altered in these diseases.

Prosomatostatin-derived peptides are widely distributed in the brain (Patel et al., 1981; Benoit et al., 1982, 1985; Lechan et al., 1983). Previous immunohistochemical studies, using antisera directed against somatostatin 28 (SS-28; an N-terminal extension of somatostatin 14) or somatostatin $28_{1-12}$ (SS-28 $8_{1-12}$; the $\mathrm{N}$-terminal dodecapeptide of SS-28), revealed an extensive neuronal and fiber system in the hippocampal formation of the rat and monkey (Morrison et al., 1982; Bakst et al., 1985, 1986). While somatostatin-immunoreactive cells and fibers were seen in each of the fields that constitute the hippocampal formation (which comprises several distinct cytoarchitectonic fields, including the dentate gyrus, the hippocampus proper, the subicular complex, and the entorhinal cortex), the dentate gyrus was particularly impressive because of the large number of immunoreactive cells and fibers and the similarity of the distribution of these elements in the 2 species. The somatostatin-immunoreactive cells in the dentate gyrus were located mainly in the polymorphic layer (the hilus), and the densest fiber and terminal labeling were observed in the outer two-thirds of the molecular layer (Morrison et al., 1982; Bakst et al., 1985). Further experimental studies indicated that the fibers in the molecular layer arise largely from somatostatin-immunoreactive cells located in the hilus and do not originate significantly in the entorhinal cortex (which contributes the major portion of the non-somatostatin fibers and terminals in this region of the dentate gyrus) (Bakst et al., 1986).

One of the great advantages of employing immunohistochemical techniques in neuroanatomy is the potential for comparing the organization of identified systems in different species; in particular, this methodology allows the nonexperimental analysis of neuronal systems in the human brain. Since the somatostatin system of the dentate gyrus has been well characterized in the rat and monkey, we sought to determine whether the organization of this system was similar in the human dentate 
gyrus. While a number of technical difficulties might potentially have hampered this investigation, we have been able to reliably stain a system of somatostatin-immunoreactive fibers and cell bodies in the human dentate gyrus that closely resembles that observed in experimental animals.

\section{Materials and Methods}

\section{Brain material}

Specimens of human brain material were obtained from different sources, and, in each case, the fixation procedure was somewhat different. For this reason, we will describe the preparative procedures for the 4 cases from which most of our observations were obtained. In all cases, the postmortem material was obtained from patients who were neurologically normal.

$C-2-86$. This brain was obtained from a 64 -year-old female who died from metastatic breast carcinoma. The brain was removed $30 \mathrm{~min}$ after death, and perfusion fixation was initiated $2 \mathrm{hr}$ later. Solutions were introduced bilaterally through the carotid arteries. Perfusion was initiated with 2 liters of $0.9 \% \mathrm{NaCl}$, followed by 4 liters of $4 \%$ paraformaldehyde in $0.1 \mathrm{~m}$ phosphate buffer. The brain, suspended by ligatures through the basilar artery, was placed in toto in 4 liters of the same fixative at $4^{\circ} \mathrm{C}$ for $3 \mathrm{~d}$. It was then placed in a solution of $20 \%$ glycerol in $0.1 \mathrm{M}$ phosphate buffer for $4 \mathrm{~d}$, then cut into $1 \mathrm{~cm}$ blocks and returned to the glycerol solution for $7 \mathrm{~d}$. The blocks of brain tissue were then frozen and stored at $-70^{\circ} \mathrm{C}$. Sections $50 \mu \mathrm{m}$ thick were cut through the temporal lobe on the freezing microtome and a 1 -in-10 series of sections was processed as described below for the demonstration of somatostatin immunoreactivity.

HC87-7. This brain was obtained from a 62-year-old male who died of a myocardial infarction. The brain was removed $1 / 2 \mathrm{hr}$ after death and placed in chilled $\left(4^{\circ} \mathrm{C}\right)$ PBS. One hour later, the brain was cut into $1 \mathrm{~cm}$ coronal blocks and placed into a periodate-lysine-paraformaldehyde fixative solution, with the final concentration of paraformaldehyde being 2\% (McLean and Nakane, 1974). After $48 \mathrm{hr}$ of fixation, the tissue blocks were taken through a graded series of sucrose solutions to $18 \%$ sucrose in PBS over a $2 \mathrm{~d}$ period. For this study, a tissue block from the mid-rostrocaudal level of the hippocampal formation was frozen on dry ice, sectioned at $40 \mu \mathrm{m}$ on a cryostat, and a 1 -in- 10 series of sections was processed for immunohistochemistry.

$S C 87-26$. This brain was obtained from a 49-year-old female who died of metastatic breast carcinoma. The brain was fixed after a postmortem interval of $8 \mathrm{hr}$ by perfusion through the internal carotid and basilar arteries. Three liters of chilled $\left(4^{\circ} \mathrm{C}\right) 4 \%$ paraformaldehyde in PBS were perfused over $45 \mathrm{~min}$. The brain was then suspended by the basilar artery in the same fixative for $12 \mathrm{hr}$. Subsequently, the brain was cut into $1 \mathrm{~cm}$ coronal blocks and postfixed in the same fixative for an additional $48 \mathrm{hr}$. A tissue block similar to that for HC87-7 was obtained and processed in a similar fashion.

CH86-3. This brain was obtained from an 82-year-old female who died of respiratory failure resulting from complications of pneumonia and congestive heart failure. The brain was removed after a postmortem interval of $8 \mathrm{hr}$, cut into $1 \mathrm{~cm}$ coronal blocks, and placed in chilled $\left(4^{\circ} \mathrm{C}\right) 4 \%$ paraformaldehyde in PBS for $72 \mathrm{hr}$. Tissue was selected and processed as in the previous case.

\section{Immunohistochemical processing}

As in our previous studies in the rat and monkey, 2 primary antisera were used in our initial studies in the human brain. (These antisera were kindly donated by Dr. Robert Benoit, McGill University.) A description of the production and specificity of these antisera has been given in our previous publications (Bakst et al., 1985, 1986; Lewis et al., 1986; Campbell et al., 1987) and references therein. In short, antiserum S320 has an antigenic determinant that corresponds to the last 8 amino acids of SS-28 $8_{1-12}$ and reacts exclusively with SS-28 $8_{1-12}$. Antiserum S309 is directed against the first 14 amino acids of SS- 28 and reacts predominantly with SS-28, with slight cross-reactivity with SS-28 ${ }_{1-12}$. In studies in the rat and monkey, antiserum S320 preferentially demonstrates fiber systems in the hippocampal formation, whereas antiserum S309 reveals a much larger population of stained neuronal cell bodies. In the human hippocampal formation, differential staining with these antisera was not as pronounced as in the rat and monkey. In particular, antiserum S320 appeared to demonstrate neurons equal in number to those in the preparations incubated with antiserum $\$ 309$, and the neurons tended to be more completely stained with $\mathbf{S 3 2 0}$ (with staining extending into distal dendritic processes). Labeled fibers and terminals were also well stained in the $S 320$ preparations. Thus, the bulk of our material was processed only with antiserum S320, and all of the observations reported in this paper are taken from these preparations. The present description of the distribution of somatostatin immunoreactivity in the human dentate gyrus is based, therefore, on the detection of $\mathbf{S S}_{1-12}$.

Free-floating sections from each of the brains were processed according to the avidin-biotin method of Hsu et al. (1981), using Vectastain kits. To reduce endogenous peroxidase activity, the tissue was first placed in a $1.0 \%$ solution of $\mathrm{H}_{2} \mathrm{O}_{2}$ for $10 \mathrm{~min}$. After washing in PBS (pH 7.4), the sections were incubated for $48 \mathrm{hr}$ at $4^{\circ} \mathrm{C}$ with either immune serum S309 at a dilution of 1:3000 or immune serum S320 at a dilution of 1: 4000 , containing $0.05 \%$ bovine serum albumin and $0.3 \%$ Triton X-100. Sections were then incubated in biotinylated goat anti-rabbit antiserum and incubated in avidin-biotin complex. Sections were developed by treatment with a chromogen solution of $0.05 \%$ diaminobenzidine (DAB) containing $0.03 \% \mathrm{H}_{2} \mathrm{O}_{2}$ for approximately $20 \mathrm{~min}$. The staining was intensified by placing the mounted and defatted sections in a solution of $0.005 \% \mathrm{OsO}_{4}$ for $30 \mathrm{~min}$, followed by a $0.05 \%$ solution of thiocarbohydrazide for $15 \mathrm{~min}$. The sections were then returned to the $\mathrm{OSO}_{4}$ solution for $30 \mathrm{~min}$. The sections were finally washed, dehydrated, and coverslipped with DPX mountant. A more detailed description of this procedure is presented in Bakst et al. (1985).

In all cases, either adjacent sections or sections close to those processed immunohistochemically were stained by the Nissl method. Selected immunohistochemically processed sections were counterstained with a modification of the Giemsa method (Iniguez et al., 1985).

\section{Analysis}

Immunohistochemically stained material was analyzed with bright- and dark-field optical systems on a Leitz Dialux-20 microscope, and highmagnification photomicrographs were taken on this system. Additional photographs were taken on a Zeiss 405 inverted microscope. Low-power photomicrographs were taken with a Nikon Multiphot system with a Nikon BD-2 dark-field base. To compare the size of somatostatin-immunoreactive cells in the human dentate gyrus with that of those previously quantified from the monkey, samples of 100 cells each from case $\mathrm{C}-2-86$ and case $\mathrm{HC} 87-7$ were measured using computer quantification of video images of labeled neurons (Young et al., 1985). Equivalent diameters of the cells and the area of the somata were analyzed.

\section{Results}

\section{General appearance of stained material}

The distribution of somatostatin-immunoreactive cells and fibers in the human dentate gyrus was similar in each of the brains examined. While staining tended to be somewhat more robust in the brains with shorter postmortem intervals to fixation, the general pattern of staining was consistent in all cases. In the

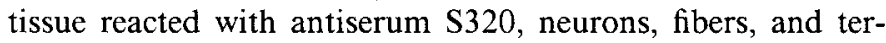
minal plexuses were clearly stained (Figs. 1-3). The density of staining of immunoreactive neurons varied greatly. In some, the precipitate had a particulate appearance (see Fig. $5 \mathrm{~A}$ ) and did not extend far into the dendrites. Other stained neurons contained dense precipitate (Fig. 3, $A, B$ ) which extended into the dendrites and gave the cells a Golgi-like appearance. Somatostatin-immunoreactive axonal profiles were more varied in thickness in the human dentate gyrus than in the rat or monkey. Regions of presumed terminal distribution, such as the outer two-thirds of the molecular layer, demonstrated both distinct axonal profiles, many of which had a beaded appearance, and a fine and relatively homogenous particulate staining. As we will describe below, there were a number of stained profiles in the human dentate gyrus that were not observed in the rat or monkey. This fact, coupled with the variability in thickness of stained axonal profiles and the varied morphology of stained neurons, gave the staining in the human dentate gyrus a more heterogeneous appearance than that observed in either the rat 

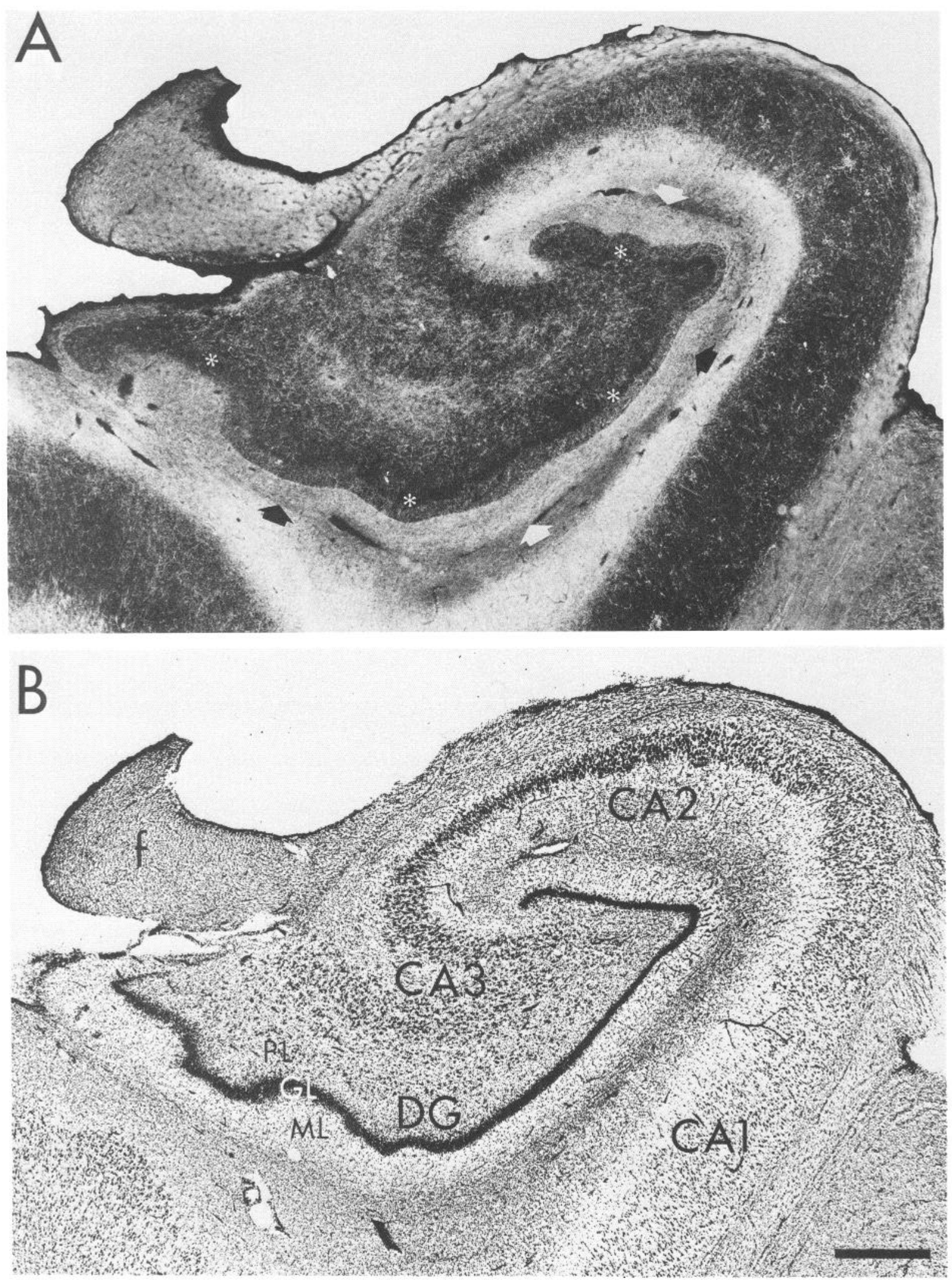

Figure 1. Low-power dark-field and bright-field photomicrographs of closely spaced coronal sections of the human dentate gyrus and hippocampus stained $(A)$ immunohistochemically, for the demonstration of SS-28 $8_{1-12}$, with antiserum S320 or $(B)$ with the Nissl method. In $B$, the 3 layers of the dentate gyrus $(D G)$ are indicated and include the molecular layer $(M L)$, the granule cell layer $(G L)$, and the polymorphic layer $(P L)$ or hilus of the dentate gyrus. The hippocampus proper is made up of 3 distinct fields: CA3, CA2, and CA1; the fimbria $(f)$ is also indicated. In $A$, regions of somatostatin fiber and terminal immunoreactivity appear as white areas. The densest labeling occurs in the outer two-thirds of the molecular layer of the dentate gyrus (bold arrows; the granule cell layer is marked with asterisks). Other regions of heavy labeling include the CA2 field and the superficial portion (stratum lacunosum-moleculare) of all hippocampal fields. Case HC87-7. Bar, $1 \mathrm{~mm}$. 

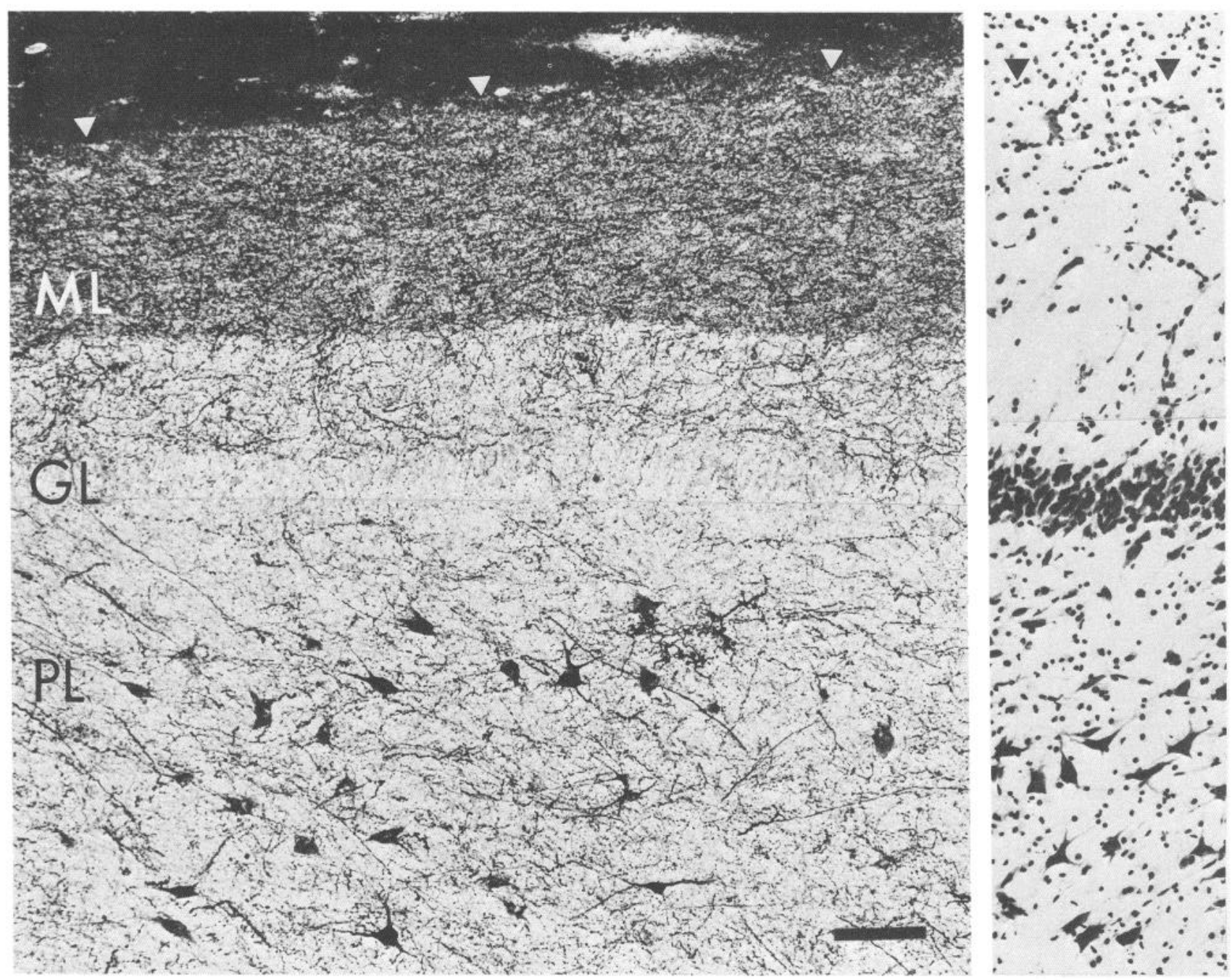

Figure 2. Higher-magnification bright-field photomicrographs of the dentate gyrus. The tissue at left has been processed for SS-28 $8_{1-12}$ immunoreactivity, and the tissue at right shows a closely spaced section stained with cresyl violet. The heaviest fibers and terminal labeling occur in the outer portion of the molecular layer $(M L)$, though there are also a moderate number of fibers in the inner third of the molecular layer. There are few somatostatin-immunoreactive fibers in the granule cell layer $(G L)$. The polymorphic layer $(P L)$ is bilaminate. Immediately subjacent to the granule cell layer is a relatively acellular zone. Deep to this is a region of moderate cellularity, and it is in this region that most of the somatostatinimmunoreactive neurons are located. There is also a variety of sizes of somatostatin-immunoreactive processes in the polymorphic layer. Case HC87-7. Bar, $100 \mu \mathrm{m}$.

or monkey. In the monkey, we observed substantial differences in the organization of the somatostatin system at different rostrocaudal levels of the hippocampal formation. Sections through the full rostrocaudal extent of the human dentate gyrus were only available from brain C-2-86, and there were no clear-cut topographic differences in the distribution of somatostatin immunoreactivity in this case. Thus, the description provided below appears to be generally applicable to all levels of the human dentate gyrus.

\section{Distribution of stained fibers and terminals}

Somatostatin-immunoreactive fibers and terminals were observed in all layers of the dentate gyrus (Figs. 1, 2). As in the rat and monkey (Fig. 6), the most densely stained plexus was located in the outer two-thirds of the molecular layer (Figs. 1, 2). Staining in this region partially consisted of labeled varicose axonal segments, many of which traveled parallel to the granule cell layer. The major component of the staining, however, was finely granular, with little indication of intervaricose axonal segments. This pattern of staining had the appearance of a diffuse terminal plexus made up of small, spherical varicosities. The inner third of the molecular layer had at least as many stained axonal profiles as did the outer two-thirds of the layer (Fig. 2). Many of these processes were oriented perpendicular to the granule cell layer, but others were obliquely oriented. The density of fibers stained in this region appeared to be substantially higher than in the rat and monkey (see Fig. 6). The strikingly lighter staining in this portion of the molecular layer is accounted for by the nearly complete absence of the finely granular staining found in the outer molecular layer. The lowest density of fiber and terminal staining occurred in the granule cell layer. Fibers passed mainly perpendicularly to the cell layer as they traveled between the hilus and the molecular layer.

Fiber labeling in the hilus produced a rather heterogeneous meshwork in which it was often difficult to differentiate labeled axonal profiles from the numerous stained dendrites. There were labeled fibers of different calibers and many contained apparent terminal varicosities. In some cases, the terminal varicosities 

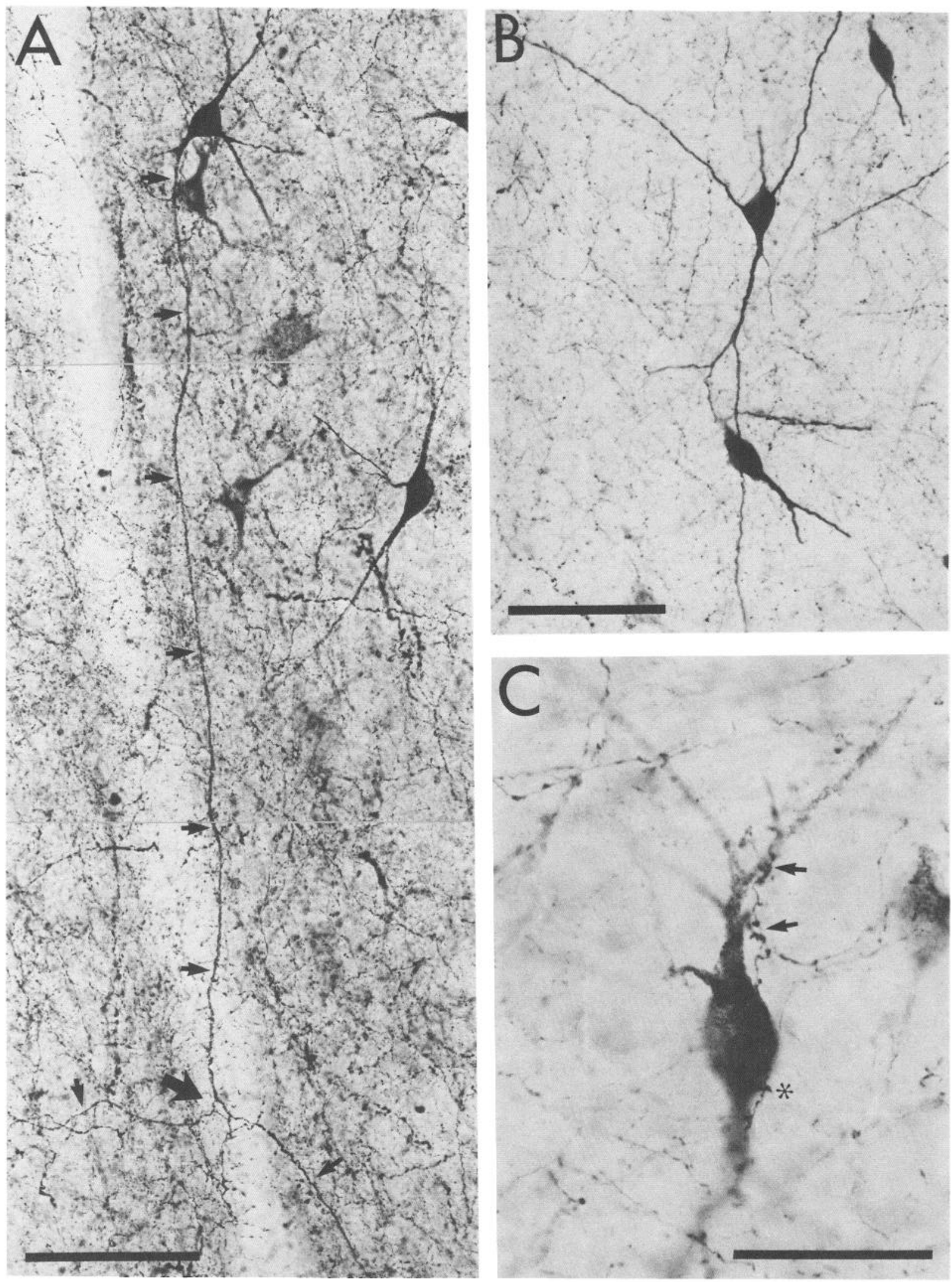

Figure 3. $A-C$, Higher-magnification bright-field photomicrographs of several somatostatin-immunoreactive neurons. While most are multipolar, they vary greatly in size and shape. $A$, An immunoreactive process (arrows) extends for nearly $700 \mu \mathrm{m}$ under the granule cell layer before breaking up (large arrow) into what appears to be a terminal ramification. In $C$, a fine, immunoreactive fiber gives rise to varicosities that are closely apposed to a somatostatin-immunoreactive neuron. Case C-2-86. Bars: $100 \mu \mathrm{m}(A, B) ; 50 \mu \mathrm{m}(C)$. 

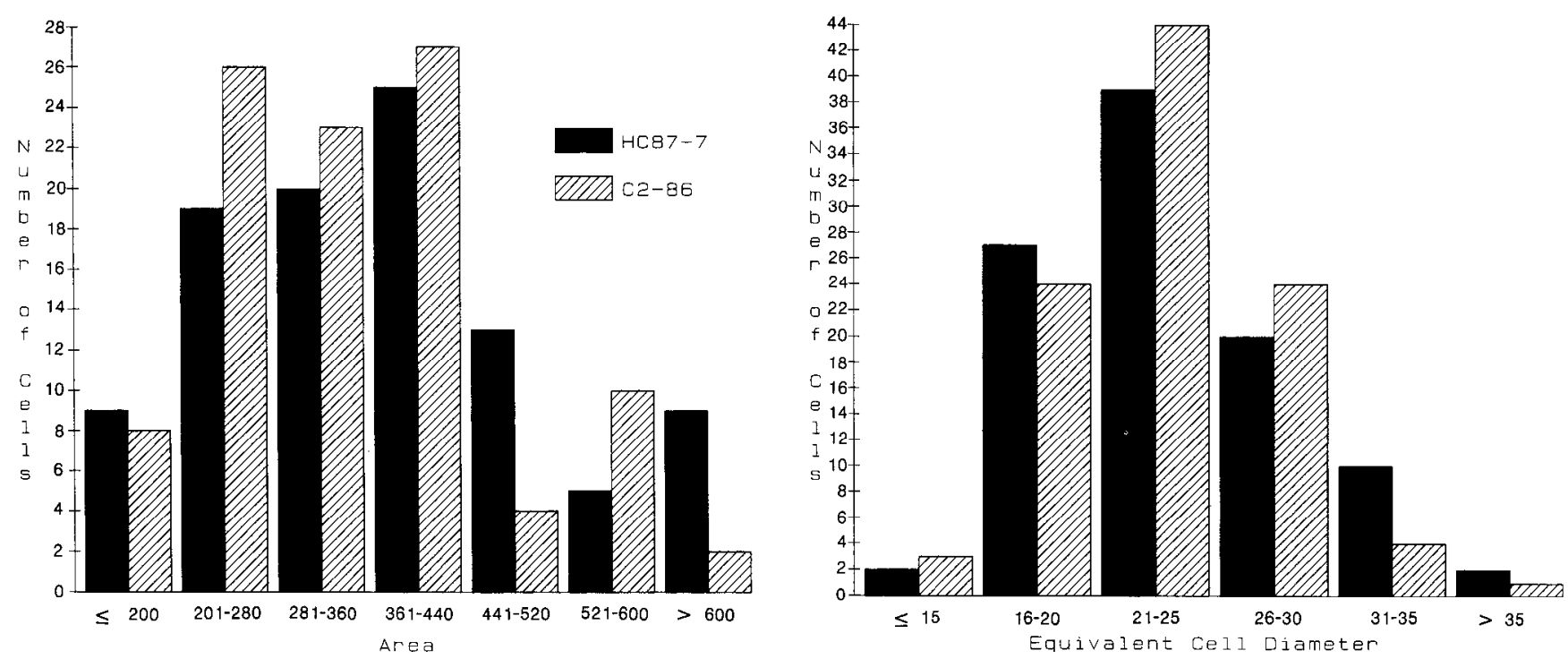

Figure 4. Histograms showing the distribution of measured areas (left) or equivalent diameters (right) of somatostatin-immunoreactive cells sampled from the hilar region of cases C-2-86 and HC87-7. One hundred cells were measured in each case, and measurements are in $\mu \mathrm{m}^{2}$ for areas and $\mu \mathrm{m}$ for equivalent diameters.

were observed to be in close proximity to somatostatin-immunoreactive cell bodies (Fig. $3 C$ ). In other cases, the terminal ramifications of these fibers formed pericellular basket plexuses that outlined the unstained ghosts of hilar neurons. In general, the somatostatin-immunoreactive fibers did not demonstrate a dominant orientation, although many collected at the medial and lateral angles of the hilus, where they appeared to pass through the granule cell layer and enter the molecular layer.

\section{Morphology and distribution of somatostatin-immunoreactive neurons}

As in the rat and monkey, there were few somatostatin-immunoreactive neurons observed in the molecular layer or in the granule cell layer. In the polymorphic layer, however, there were numerous stained cells that varied greatly in size and shape. The primate polymorphic layer is bilaminate; immediately below the granule cell layer, there is a paucicellular region in which few somatostatin-positive cells were seen. Deep to this region is where most of the hilar neurons are located (Fig. 2), and many of these are somatostatin-immunoreactive. The somatostatinpositive neurons ranged from small, round cells to large, multipolar cells (Figs. 2,3). Some of the cells had a distinctly fusiform shape, with prominent dendrites emanating from 2 poles. A number of these cells gave rise to axons that could often be seen to ramify locally, but few axons were followed for long distances. In some cases, neurons gave rise to long, thick processes that extended for several hundred microns before breaking up into an apparent terminal plexus (Fig. $3 A$ ). The sizes, shapes, and distribution of somatostatin-labeled cells remained similar throughout the rostrocaudal extent of the dentate gyrus.

In the macaque monkey, the mean area of hilar somatostatin cells is $164.72 \mu \mathrm{m}^{2}\left(\mathrm{SD}=55 \mu \mathrm{m}^{2}\right)$, with a range of $42.65-316.79$ $\mu \mathrm{m}^{2}$. In the samples measured from the human brains (Fig. 4), the cells were found to be substantially larger than in the monkey. The mean area for the cells sampled in case C-2-86 was $346.12 \mu \mathrm{m}^{2}$, with a range of $141-754 \mu \mathrm{m}^{2}$; the mean area of the cells sampled from case $\mathrm{HC} 87-7$ was $379.73 \mu \mathrm{m}^{2}(\mathrm{SD}=152.51$ $\mu \mathrm{m}^{2}$ ), with a range of $138-892 \mu \mathrm{m}^{2}$. The mean equivalent di- ameter of hilar somatostatin cells in the monkey was found to be $18.12 \mu \mathrm{m}(\mathrm{SD}=3.74 \mu \mathrm{m})$, with a range of $8.97-28.72 \mu \mathrm{m}$. In human case C-2-86, the mean equivalent diameter was 22.74 $\mu \mathrm{m}(\mathrm{SD}=4.55 \mu \mathrm{m})$, with a range of 13.9-36.1 $\mu \mathrm{m}$, and for case HC87-7 the mean was $23.28 \mu \mathrm{m}(\mathrm{SD}=5.08 \mu \mathrm{m})$, with a range of $14.2-36.6 \mu \mathrm{m}$.

\section{Somatostatin-immunoreactive profiles not previously observed in the rat and monkey}

While the general distribution of somatostatin immunoreactivity is similar in the human and nonhuman dentate gyrus, there are a number of features seen in the human brain that were not formerly observed in the rat or monkey hippocampal formation. Perhaps the most striking profiles of this type were clusters of large, grapelike varicosities that were seen throughout the hippocampal formation and prominently in the hilus of the dentate gyrus (Fig. 5, $A, B$ ). These profiles were observed in all 4 of the cases studied and had a consistent appearance. In each case, a darkly stained varicose fiber would ramify extensively in a small arca, giving rise to strings of collatcrals bearing large varicositics (Fig. $5 B$ ). The staining of many of the larger varicosities was often inhomogeneous, and unstained vacuoles could be observed within them.

We also commonly observed long, darkly stained dendritic profiles that contained clusters of what appeared to be dendritic spines (Fig. 5, C, D). In some cases the spines had a typical appearance (Fig. $5 C$ ), whereas in others the spines were longer and thicker than typical spines (Fig. $5 D$ ). There were also a number of stained profiles in the hilus that did not closely resemble either typical axonal or dendritic profiles. The stained profile in Figure $5 E$, for example, is thick and extends from the hilus into the molecular layer, where it appears to break up into a terminal plexus. This process is clearly thicker than other axons in the field, however, and there are thin profiles along the trajectory of the stained process that resemble dendritic spines. It is difficult, thercforc, to label this process as axonal or dendritic. 

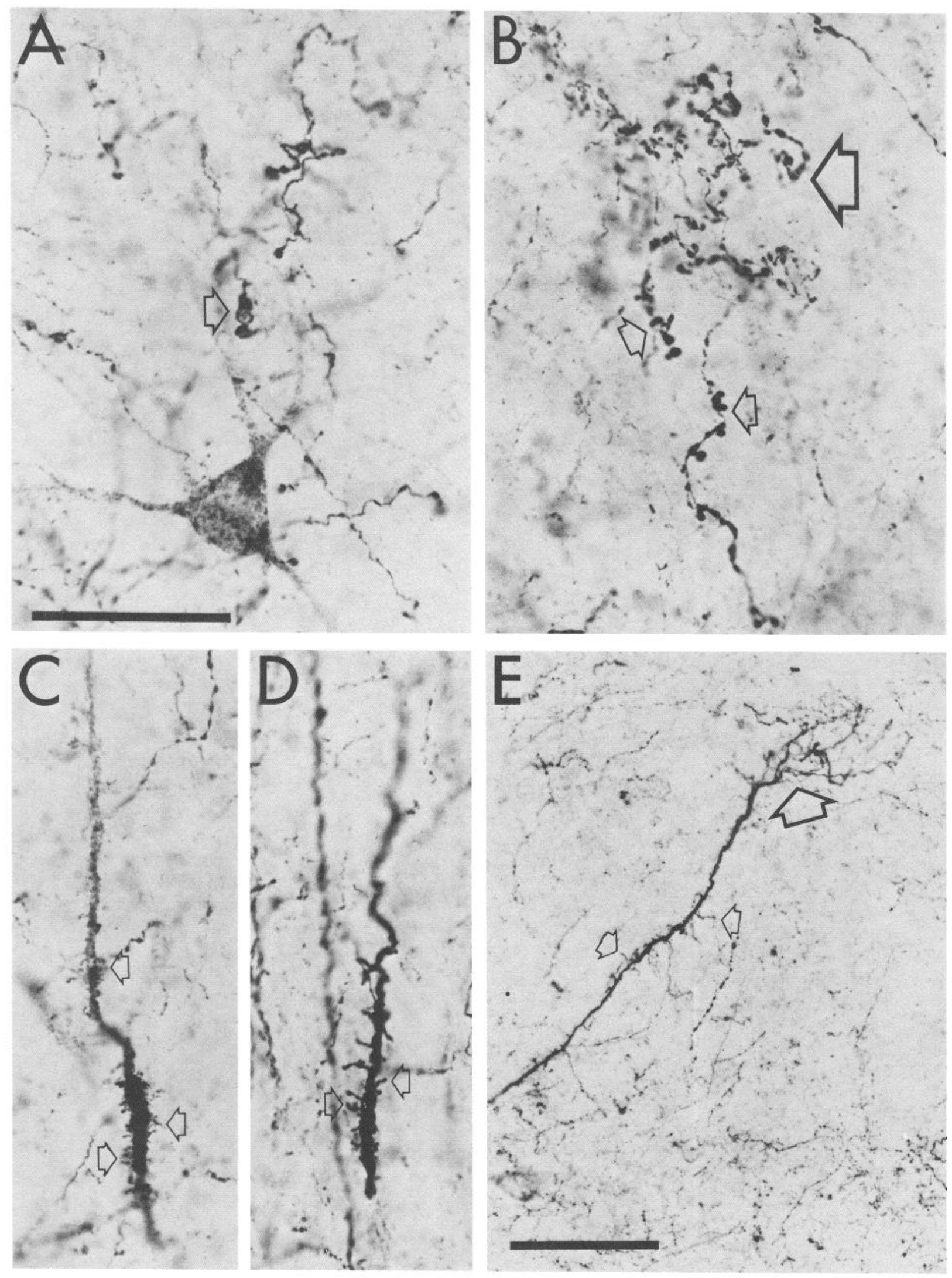

Figure 5. A variety of stained processes observed in the human dentate gyrus was not previously observed in the rat or macaque monkey. $A$, Large, immunoreactive varicosities (arrow) are seen in the vicinity of a stained neuron. These varicosities are often observed in clusters like the one pictured in $B$. $C, D$, Photomicrographs show immunoreactive profiles that resemble dendrites with dendritic spines (arrows). Certain of the immunoreactive profiles were difficult to classify. The profile in $E$, for example, appears to generate a terminal ramification in the molecular layer (large arrow) and might be considered an axon, but the fine processes along the trajectory of the profile (small arrows) appear more like dendritic spines. Case C-2-86 $(A-D)$, HC87-7 $(E)$. Bars: $50 \mu \mathrm{m}(A-D) ; 100 \mu \mathrm{m}(E)$. 

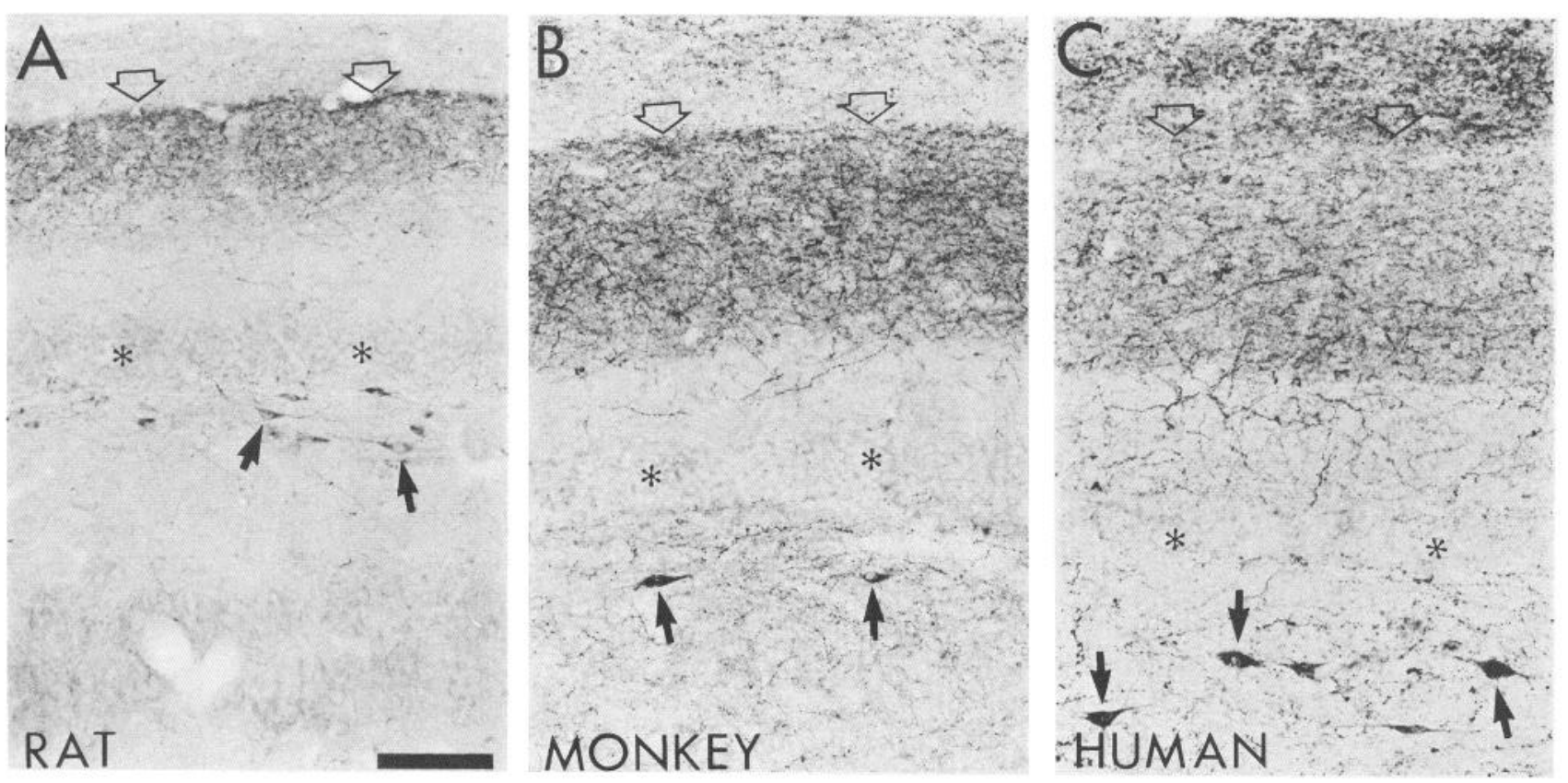

Figure 6. Bright-field photomicrographs through the dentate gyrus of the rat $(A)$, macaque monkey $(B)$, and human $(C)$ brains photographed at the same magnification. The hippocampal fissure is indicated by open arrows in $A-C$ and the dark band just below the fissure is indicative of somatostatin fiber and terminal labeling. The granule cell layer has been indicated with asterisks. Note that staining in the inner third of the molecular layer is somewhat more prominent in the human than in the other species. Somatostatin-immunoreactive neurons are indicated with arrows in the polymorphic layer. Human case C-2-86. Bar, $100 \mu \mathrm{m}$.

\section{Discussion}

In a series of previous papers, the distribution of somatostatinimmunoreactive neurons and fibers in the hippocampal formation was described for the rat and monkey brain (Morrison et al., 1982; Bakst et al., 1985, 1986). In both species, the most distinctive staining was located in the dentate gyrus, where a heterogeneous population of somatostatin-immunoreactive neurons was observed in the polymorphic layer of the hilar region and a dense terminal plexus was seen in the outer twothirds of the molecular layer. Subsequent experimental studies, employing either selective ablation of the entorhinal cortex (the origin of the major projection to the outer molecular layer) or kainic acid destruction of the cells of the hilar region, indicated that the fiber plexus originates almost exclusively from the cells of the ipsilateral hilar region. Recent axonal tracing studies $(\mathrm{K}$. Krzemieniewska and D. G. Amaral, unpublished observations) using the lectin PHA-L have confirmed that cells of the hilar region do indeed give rise to fibers that terminate in the outer two-thirds of the molecular layer, and that the projection is both relatively local (i.e., is not divergent in the long or septotemporal axis) and quite distinct from the well-known associational projection to the inner third of the molecular layer (which does not arise from the somatostatin-immunoreactive hilar cells).

In the present study, we sought to determine whether the human dentate gyrus contained a somatostatin system similar to that in the rat and monkey. In order to have tissue comparable to that in the rat and monkey, we have used the same antisera and histological procedures that were used previously. The largest deviation from practices used in the experimental animals was in the fixation protocols. While several fixation procedures were used, the somatostatin staining appeared to be qualitatively similar in all cases. The shortest postmortem interval to fixation was in case $\mathrm{HC} 87-7$, in which immersion fixation was initiated approximately $1 \frac{1 / 2}{\mathrm{hr}}$ after death. Perfusion fixation was initiated in case C-2-86 some $2 \frac{1}{2} \mathrm{hr}$ after death. While the staining in these 2 cases was more robust than in the other cases, in which fixation was initiated some $8 \mathrm{hr}$ after death, all components of the somatostatin system were also apparent in these latter cases. Moreover, no obviously aberrant staining was observed in the latter cases that might be indicative of postmortem degradation.

The distribution of somatostatin immunoreactivity in the dentate gyrus of the rat, macaque monkey, and human is shown in Figure 6. It is clear that the overall organization of this system is similar in the 3 species. All have a heterogeneous population of labeled cells in the hilar region and a band of immunoreactive fibers and terminals in the outer two-thirds of the molecular layer. Subtle differences in the organization of the somatostatin system had already been detected, however, in comparisons of the rat and monkey brain. For example, it had previously been noted that the density of fiber and terminal labeling was greater in the molecular layer of the monkey than of the rat. In the current study, we observed that the staining of somatostatin fibers and terminals in the human molecular layer is as strong as that of the monkey, confirming our suggestion that the somatostatin system may be more prominent in the primate hippocampal formation than in the rodent. We also noted in the current study that the number of fibers labeled in the inner third of the human molecular layer was substantially higher than that observed in the monkey. Perhaps the most significant difference in the overall staining pattern in the human dentate gyrus was the greater labeling of neuronal cell bodies with antiserum S320. This antiserum, which is specific for SS-28 $8_{1-12}$, stained far fewer neurons in the rat and monkey than antiserum S309, which is directed primarily against SS-28. In the human, however, incubation with antiserum S320 led to the staining of equal or 
greater numbers of neurons, and the staining of the neurons tended to be more complete. Whether this finding reflects a change in the proportion of prosomatostatin peptides in neurons of the human hippocampus or perhaps a postmortem breakdown of SS-28 to SS-28 $8_{1-12}$ cannot be determined from current information.

The types of labeled cells in the hilar region of the 3 species appeared to be similar. In each case, the population of labeled cells contained neurons of various sizes and shapes. Labeled cells in the human hilar region ranged in somal area from approximately $140 \mu \mathrm{m}^{2}$ to nearly $900 \mu \mathrm{m}^{2}$, and were thus somewhat larger than those in the monkey, which had a range from 43 to $317 \mu \mathrm{m}^{2}$. The large variability of the sizes of labeled cells reflects the neuronal heterogeneity in the hilar region (Amaral, 1978) and the likelihood that somatostatin immunoreactivity is an attribute of several neuronal cell types.

Chan-Palay (1987) has recently reported on the distribution of somatostatin immunoreactivity in the human hippocampal formation. Using different antisera and staining procedures from those in this study, she reported a distribution of staining in the dentate gyrus that is mainly consistent with our findings. In particular, she noted that the largest number of labeled cells was located in the hilar region, and that there was a plexus of stained fibers in the outer portion of the molecular layer. Also consistent with the dense somatostatin fiber and terminal labeling in the outer portion of the molecular layer is the high density of somatostatin receptors observed there by Reubi et al. (1986).

While the overall pattern of staining in the human dentate gyrus was similar to that in the rat and monkey, there were forms of stained profiles that had not been previously observed in the experimental animals. In particular, large, grapelike clusters of apparent axonal varicosities were commonly observed in the hilus of the dentate gyrus. Interestingly, these were not observed in the granule cell or molecular layers. Similar profiles were common in the other fields of the hippocampal formation and were particularly numerous in the CAl field. Chan-Palay (1987) has reported observing similar somatostatin-immunoreactive profiles in the human hippocampal formation. Wc also observed a variety of stained dendritic processes that bore apparent dendritic spines. In the preparations previously studied from the rat and monkey, we rarely observed stained spines.

The distribution of a number of other transmitter-specific systems has recently been analyzed in the monkey and human hippocampal formation. In a study of the distribution of neuropeptide $Y$ in the monkey, Kohler et al. (1986) reported a system of immunoreactive cells and fibers that was very similar to that observed for somatostatin in the monkey (Bakst et al., 1985). In a subsequent study of the human hippocampal formation, Chan-Palay et al. (1986a, b) reported a distribution of neuropeptide $\mathrm{Y}$-immunoreactive cells similar to the one they had observed previously and to that described in the present study for somatostatin. In contrast both to their previous findings in the monkey and to the high density and laminar organization of the somatostatin fibers observed in the present study, however, Chan-Palay and colleagues reported "radial varicose fibers scattered throughout (the dentate gyrus) without a clear laminar preference." In agreement with our observations, though, they also reported the occurrence of neuropeptide $Y$-immunoreactive grapelike clusters of axonal varicosities that were similar to those stained for somatostatin in the present study. Chan-Palay (1987) has recently indicated, in fact, that there is substantial colocalization of neuropeptide $\mathrm{Y}$ and somatostatin in the cells of the human hilar region. Del Fiacco et al. (1987) have studied the distribution of substance $P$ immunoreactivity in the human hippocampal formation and found in the dentate gyrus that there are many substance P-positive cells in the hilus, whereas labeled fibers are located either immediately above and below the granule cell layer or, somewhat more heavily, in the outer two-thirds of the molecular layer. Finally, Schlander et al. (1987) have demonstrated a distribution of glutamic acid decarboxylase (GAD)-containing neurons and fibers in the human dentate gyrus similar to that observed in experimental animals. Most of the neurons were visualized in the hilus or on the interface between the hilus and the granule cell layer, and most of the immunoreactive terminals were observed in the granule cell layer. In nonhuman mammals, many neurons in the hippocampal formation colocalize GAD and somatostatin (Schmechel et al., 1984), although the extent of this overlap in the hilar region is not yet clear. Taken together, these studies indicate that the human dentate gyrus contains the same transmitter systems observed in experimental animals, and that immunohistochemical methodologies allow the comparison of the organization of these systems in a variety of species.

Since the functions of the somatostatin system in the hippocampal formation are not known at present, and the physiological effects of somatostatin release remain controversial, we will refrain from discussing the possible roles of this transmitter in the normal functioning of the human hippocampus. We should point out, however, that alterations in somatostatin function have been linked to at least 2 pathological conditions.

Work by Ball $(1977,1978)$ and others (e.g., Corsellis, 1970; Hooper and Vogel, 1976; Hyman et al., 1984, 1986) has demonstrated that the hippocampal formation is severely damaged in Alzheimer's disease. Ball (1978) found that the entorhinal cortex contained the highest number of neurofibrillary tangles, followed in order by the subiculum, CA1, CA3, presubiculum, and CA2. Granulovacuolar degenerations were most common in the subiculum, followed in order by CA1, CA2, CA3, entorhinal cortex, and the presubiculum. The dentate gyrus was considercd to be free of both of these markers. Hyman and colleagues $(1984,1986)$ confirmed many of the findings of Ball (1978) and reported that the outer two-thirds of the molecular layer of the dentate gyrus contained a high density of neuritic plaques. More recently, and in contrast to Ball (1978), ChanPalay (1987) has noted a high incidence of neurofibrillary tangles and neuritic plaques in the hilus of the dentate gyrus.

Somatostatin appears to be one of the neuropeptide systems that is adversely affected in Alzheimer's disease. Biochemical studies have shown that there is both a decrease in somatostatin content (Davies et al., 1980; Rossor et al., 1980; Davies and Terry, 1981; Beal et al., 1986) and receptors (Beal et al., 1985) in the hippocampal formation and neocortex. Although there have been limited morphological studies of the hippocampus, studies in the neocortex have shown that somatostatin-positive neuritic processes are found in neuritic plaques (Armstrong et al., 1985; Morrison et al., 1985; Nakamura and Vincent, 1986). Nakamura and Vincent (1986), despite finding significant abnormalities in somatostatin-positive neurites, found no appreciable loss of somatostatin neurons in the neocortex of Alzheimer's disease tissue. Roberts et al. (1985), however, reported both decreases in somatostatin-immunoreactive profiles and the occurrence of somatostatin-immunoreactive neurofibrillary tangles in the neocortex. They also reported that somatostatinimmunoreactive neurofibrillary tangles and dystrophic somato- 
statin-immunoreactive neurons were present in the hippocampus. Chan-Paley (1987) has suggested that the most severe depletion of somatostatin-immunoreactive profiles in the hippocampal formation occurred in the hilus of the dentate gyrus. Because the dentate gyrus provides the main relay of cortical information entering through the entorhinal cortex to the other fields of the hippocampal formation, it is likely that any significant adverse effects of Alzheimer's disease on the somatostatin system in the dentate gyrus would compromise the ability of the hippocampal formation to function properly. Since damage to the hippocampal formation has been associated with deficits in declarative memory (Zola-Morgan et al., 1986), disruption of the somatostatin system may contribute to the impairment of memory function associated with Alzheimer's disease.

Alterations in the hippocampal somatostatin system have also recently been associated with the pathology of temporal lobe epilepsy. Seizure activity in the hippocampal formation has been implicated as a primary factor in the pathogenesis of temporal lobe epilepsy, the most common form of the disease (Meldrum and Corsellis, 1985). While it is beyond the scope of this paper to discuss the various causative factors of temporal lobe epilepsy, it is of interest to note that Sloviter (1987) has observed a relatively selective loss of certain classes of interneurons in the dentate gyrus of rats treated to produce an experimental model of epilepsy. In particular, somatostatin-immunoreactive cells in the hilus were nearly completely eliminated by the electrical stimulation paradigm employed, whereas other interneuronal cell types, such as the GABAergic dentate pyramidal basket cells, showed only a meager depletion. The possibility of selective loss of transmitter-specific systems in temporal lobe epilepsy is of substantial interest in determining the etiology and treatment of the disease. It would be advantageous, therefore, to determine whether human epileptic hippocampal formation demonstrates the loss of a transmitter-specific class of neurons and, in particular, whether the somatostatin system is particularly affected. The immunohistochemical methods employed in this study demonstrate the practicality of this type of neuropathological analysis, and the results of the present study provide the baseline observations with which to compare the pathologic material.

\section{References}

Amaral, D. G. (1978) A Golgi study of the cell types in the hilar region of the hippocampus of the rat. J. Comp. Neurol. 182: 851-914.

Armstrong, D. M., S. LeRoy, D. Shields, and R. D. Terry (1985) Somatostatin-like immunoreactivity within neuritic plaques. Brain Res. 338: 71-79.

Bakst, I., J. H. Morrison, and D. G. Amaral (1985) The distribution of somatostatin-like immunoreactivity in the monkey hippocampal formation. J. Comp. Neurol. 236: 423-442.

Bakst, I., C. Avendaño, J. H. Morrison, and D. G. Amaral (1986) An experimental analysis of the origins of somatostatin-like immunoreactivity in the dentate gyrus of the rat. J. Neurosci. 6: 1452-1462.

Ball, M. J. (1977) Neuronal loss, neurofibrillary tangles and granulovacuolar degeneration in the hippocampus with aging and dementia. Acta Neuropathol. 37: 111-118.

Ball, M. J. (1978) Topographic distribution of neurofibrillary tangles and granulovacuolar degeneration in hippocampal cortex of aging and demented patients. A quantitative study. Acta Neuropathol. 42: 7380.

Beal, M. F., M. F. Mazurek, V. T. Tran, G. Chattha, E. D. Bird, and I. B. Martin (1985) Reduced numbers of somatostatin receptors in the cerebral cortex in Alzheimer's disease. Science 229: 289-291.

Beal, M. F., M. F. Mazurek, C. N. Svendsen, E. D. Bird, and J. B.
Martin (1986) Widespread reduction of somatostatin-like immunoreactivity in the cerebral cortex in Alzheimer's disease. Ann. Neurol. $20: 489$.

Benoit, R., N. Ling, B. Alford, and R. Guillemin (1982) Seven peptides derived from pro-somatostatin in rat brain. Biochem. Biophys. Res. Commun. 107: 944-950.

Benoit, R., P. Bohlen, N. Ling, F. Esch, A. Baird, S. Y. Ying, W. B. Wehrenberg, R. Guillemin, J. H. Morrison, C. Bakit, L. Koda, and F. E. Bloom (1985) Somatostatin-28 $8_{1-12}$-like peptides. In Somatostatin, Y. C. Patel and G. S. Tannenbaum, eds., pp. 89-107, Plenum, New York.

Campbell, M. J., D. A. Lewis, R. Benoit, and J. H. Morrison (1987) Regional heterogeneity in the distribution of somatostatin-28 and somatostatin- $28_{1-12}$-immunoreactive profiles in monkey neocortex. J. Neurosci. 7: 1133-1144.

Chan-Palay, V. (1987) Somatostatin immunoreactive neurons in the human hippocampus and cortex shown by immunogold/silver intensification on vibratome sections: Coexistence with neuropeptide $Y$ neurons, and effects in Alzheimer-type dementia. J. Comp. Neurol. 260: 201-223.

Chan-Palay, V., C. Kohler, U. Haesler, W. Lang, and G. Yasargil (1986a) Distribution of neurons and axons immunoreactive with antisera against neuropeptide $Y$ in the normal human hippocampus. J. Comp. Neurol. 248: 360-375.

Chan-Palay, V., W. Lang, U. Haesler, C. Kohler, and G. Yasargil (1986b) Distribution of altered hippocampal neurons and axons immunoreactive with antisera against neuropeptide $Y$ in Alzheimer's type dementia. J. Comp. Neurol. 248: 376-394.

Corsellis, J. A. N. (1970) The limbic areas in Alzheimer's and in other conditions associated with dementia. In Alzheimer's Disease: $A$ Ciba Foundation Symposium, G. E. W. Wolstenholme and M. O'Connor, cds., pp. 37-50, Churchill, London.

Davies, P., and R. D. Terry (1981) Cortical somatostatin-like immunoreactivity in cases of Alzheimer's disease and senile dementia of the Alzheimer type. Neurol. Aging 2: 9-14.

Davies, P., R. Katzman, and R. D. Terry (1980) Reduced somatostatin-like immunoreactivity in cerebral cortex from cases of Alzheimer disease and Alzheimer senile dementia. Nature 288: 279-280.

Del Fiacco, M., M. C. Levanti, M. L. Diessi, and G. Zucca (1987) The human hippocampal formation and parahippocampal gyrus: Localization of substance P-like immunoreactivity in newborn and adult post-mortem tissue. Neuroscience 21: 141-150.

Hooper, W. M., and F. S. Vogel (1976) The limbic system in Alzheimer's disease. Am. J. Pathol. 85: 1-19.

Hsu, S. M., L. Raine, and H. Fanger (1981) Use of avidin-biotin peroxidase complex (ABC) in immunoperoxidase techniques: A comparison between $\mathrm{ABC}$ and unlabeled antibody (PAP) procedures. J. Histochem. Cytochem. 29: 557-580.

Hyman, B. T., G. W. Van Hoesen, A. R. Damasio, and C. L. Barnes (1984) Alzheimer's disease: Cell-specific pathology isolates the hippocampal formation. Science 225: 1168-1170.

Hyman, B. T., G. W. Van Hoesen, L. J. Kromer, and A. R. Damasio (1986) Perforant pathway changes and the memory impairment of Alzheimer's disease. Ann. Neurol. 20: 472-481.

Iniguez, C. M., J. Gayoso, and J. Carreres (1985) A versatile and simple method for staining nervous tissue using Giemsa dye. J. Neurosci. Methods 13: 77-86.

Kohler, C., L. Eriksson, S. Davies, and V. Chan-Palay (1986) Neuropeptide $Y$ innervation of the hippocampal region in the rat and monkey brain. J. Comp. Neurol. 244: 384-400.

Lechan, R. M., R. H. Goodman, M. Rosenblatt, S. Reichlin, and J. F. Habener (1983) Prosomatostatin-specific antigen in rat brain: Localization by immunocytochemical staining with an antibody to a synthetic sequence of preprosomatostatin. Proc. Natl. Acad. Sci. USA 80: 2780-2784.

Lewis, D. A., M. J. Campbell, and J. H. Morrison (1986) An immunohistochemical characterization of somatostatin-28 and somatostatin-28 $8_{1-12}$. J. Comp. Neurol. 248: 1-18.

McLean, I., and C. Nakane (1974) Periodate-lysine-paraformaldehyde fixative: A new fixative for immunoelectron microscopy. J. Histochem. Cytochem. 22: 1077-1083.

Meldrum, B. S., and J. A. N. Corsellis (1985) In Greenfield's Neuropathology, J. H. Adams, J. A. N. Corsellis, and L. W. Duchen, eds., pp. 921-950, Wiley, New York.

Morrison, J. H., R. Benoit, P. J. Magistretti, N. Ling, and F. E. Bloom 
(1982) Immunohistochemical distribution of prosomatostatin-related peptides in hippocampus. Neurosci. Lett. 34: 137-142.

Morrison, J. H., J. Rogers, S. Scherr, R. Benoit, and F. E. Bloom (1985) Somatostatin immunoreactivity in neuritic plaques of Alzheimer's patients. Nature 314: 90-92.

Nakamura, S., and S. R. Vincent (1986) Somatostatin- and neuropeptide $Y$-immunoreactive neurons in the neocortex in senile dementia of Alzheimer's type. Brain Res. 370: 11-20.

Patel, Y. C., T. Wheatly, and C. Ning (1981) Multiple forms of immunoreactive somatostatin: Comparison of distribution in neural and non-neural tissues and portal plasma of the rat. Endocrinology 109: 1943-1946.

Reubi, J. C., R. Cortes, R. Maurer, A. Probst, and J. M. Palcios (1986) Distribution of somatostatin receptors in the human brain: An autoradiographic study. Neuroscience 18: 329-346.

Roberts, G. W., T. J. Crow, and J. M. Polak (1985) Location of neuronal tangles in somatostatin neurones in Alzheimer's disease. Nature 314: 92-94.

Rossor, M. N., P. C. Emson, C. Q. Mountjoy, M. Roth, and L. L. Iversen (1980) Reduced amounts of immunoreactive somatostatin in the temporal cortex in senile dementia of the Alzheimer type. Neurosci. Lett. 20: 373-377.

Schlander, M., G. Thomalske, and M. Frotscher (1987) Fine structure of GABAergic neurons and synapses in the human dentate gyrus. Brain Res. 401: 185-189.

Schmechel, D. E., B. G. Vickrey, D. Fitzpatrick, and R. P. Elde (1984) GABAergic neurons of mammalian cerebral cortex: Widespread subclass defined by somatostatin content. Neurosci. Lett. 47: 227-232.

Sloviter, R. S. (1987) Decreased hippocampal inhibition and a selective loss of interneurons in experimental epilepsy. Science 235: 7376.

Young, W. G., J. H. Morrison, and F. E. Bloom (1985) An electronic morphometry and mapping analysis microscopy system (EMMA) for quantitative and comparative study of neural structures. Soc. Neurosci. Abstr. 11: 679 .

Zola-Morgan, S., L. R. Squire, and D. G. Amaral (1986) Human amnesia and the medial temporal region: Enduring memory impairment following a bilateral lesion limited to field CAl of the hippocampus. J. Neurosci. 6: 2950-2967. 